\title{
Morphological, structural, and optical properties of flexible Tin Oxide(II) thin film via thermal evaporation technique
}

\author{
H. S. Wasly ${ }^{1}$, M. S. Abd El-Sadek ${ }^{2}$, Sahar Elnobi ${ }^{3}$, Amr Attia Abuelwafa ${ }^{4, a}$ \\ ${ }_{1}^{1}$ Mining, Metallurgy and Petroleum Engineering Department, Faculty of Engineering, Al-Azhar University, Qena 83513, Egypt \\ 2 Nanomaterials Lab, Physics Department, Faculty of Science, South Valley University, Qena 83523, Egypt \\ 3 Physics Department, Faculty of Science, South Valley University, Qena 83523, Egypt \\ ${ }^{4}$ Nano and Thin Film Lab. Physics Department, Faculty of Science, South Valley University, Qena 83523, Egypt
}

Received: 31 May 2021 / Accepted: 4 January 2022

(C) The Author(s) 2022

\begin{abstract}
Tin oxide compounds have been highly studied due to their important properties. Tin Oxide (II) SnO compound is considered an ideal p-type conductive material, with large p-type carrier mobility. It is getting a lot of attention in next-generation electronic applications. In this work, the $\mathrm{SnO}$ thin film was deposited on the polyethylene terephthalate (PET) substrate by the thermal evaporation method. The X-ray diffraction pattern revealed the amorphous structure of the SnO/PET thin film. The purity of the $\mathrm{SnO} / \mathrm{PET}$ film was confirmed using the Raman spectrum. An atomic force microscope was carried out to investigate the topography (roughness and particle size) of the obtained $\mathrm{SnO}$ thin film. The optical band gap $E_{\mathrm{g}}^{\mathrm{Opt}}$ for SnO/PET thin film in the absorption region was estimated using Tauc's equation. The refractive index for the SnO/PET thin film was estimated at the normal dispersion range by a single oscillator model. The dielectric optical properties of the SnO/PET thin film were estimated. The calculated third-order nonlinear susceptibility $\chi^{(3)}$ and nonlinear refractive index $\mathrm{n}^{(2)}$ were in the order of $\sim 10^{-10}$ and $\sim 10^{-9}$ esu, respectively, within the photon energy range ( 0.5 to $4 \mathrm{eV}$ ). The Sheik-Bahae model was used to determine the nonlinear absorption coefficient $\beta c$ for $\mathrm{SnO} / \mathrm{PET}$ thin film. Based on these detailed results, the high nonlinear optical parameters pave the way for the probability of using the $\mathrm{SnO} / \mathrm{PET}$ in flexible optoelectronics devices.
\end{abstract}

\section{Introduction}

Oxide semiconductors have taken an increasing interest in electronic applications due to their high-power efficiency, excellent stability, optical transparency, and flexibility [1,2]. Wide-bandgap (WBG) oxide semiconductors have recently received a lot of attention due to their use in ultraviolet (UV) optoelectronic applications, and they have opened up new, unprecedented opportunities for many smart technologies and applications such as phototransistors, solar cells, touch-sensitive display technologies, optoelectronic devices, and UV emitters [3, 4]. One of the most commercial WBGs which has taken greater consideration for electronic and optoelectronic applications is $\mathrm{SnO}$ due to its large direct bandgap, p-type conductivity, and great hypothetical specific capacity $[5,6]$. The stannous ion $\mathrm{SnO}$ has significantly different physicochemical characteristics and uses than $\mathrm{SnO}_{2}$ [7]. Moreover, $\mathrm{SnO}$ is affordable, naturally abundant, has high transparency (approximately $90 \%$ ), and low resistivity $(25 \mathrm{~m} \Omega . \mathrm{cm}$ ). So, these features make $\mathrm{SnO}$ an encouraging candidate and a promising material for next-generation transparent flexible electronics [2]. Moreover, it's a good candidate for use in solar cells, electrochromic devices, and as a transparent conductive oxide (TCO) [8-11]. Furthermore, $\mathrm{SnO}$ is used as a gas sensor owing to its high sensitivity to low gas concentrations [12].

Recently, the challenge in electronics has been to develop portable, scalable, flexible, mechanically strong, easily recyclable, and low-cost products, such as transparent paper and plastic substrates (PET), because silicon substrate technologies require high process temperatures [13]. The PET substrate has been taken seriously in recent years as a substrate for semiconductor devices [14]. It can filter UV light due to its unique properties such as flexibility, transparency, lightweight, thinness, softness, mechanical strength, and high optical transparency [14-16]. PET substrates' unique advantages can be used to replace glass in a variety of display technologies, including organic light emitting, touch-screens, mobile phones, and digital cameras $[15,16]$. Due to the advantages of each SnO thin film and PET substrate, the aim of this work is to study the structure and linear and nonlinear optical characteristics of a SnO thin film deposited on a PET substrate (referred to as the SnO/PET thin film hereafter) using the thermal evaporation technique.

\footnotetext{
a e-mail: amr.abuelwafa@sci.svu.edu.eg (corresponding author)
} 


\section{Experimental}

$\mathrm{SnO}$ (II) $99.99 \%$ powder and PET substrate were acquired from Sigma-Aldrich Co. The SnO thin film (220 nm) was prepared at room temperature by thermal evaporation (Edwards type E306A-10-6 Torr). Surface characterization of the prepared SnO/PET thin film was examined by the AFM (NT-MDT, Russia). The SnO/PET thin film's XRD pattern was created using an XRD system (Shimadzu LabX XRD-6000-Japan- = 1.5408). Raman spectroscopy was conducted with an excitation laser at $532 \mathrm{~nm}$ using a Renishaw inVia Raman microscope. The transmittance $\mathrm{T}(\lambda)$ and reflectance $\mathrm{R}(\lambda)$ were recorded at normal incidence in the wavelength range (300-2500 nm) using the double beam spectrophotometer (JASCO V-670).

\section{Results and discussion}

\subsection{Structural properties of SnO/PET}

Figure 1 displays the XRD pattern of SnO/PET thin film in the $2 \theta$ range from 10 to $50^{\circ}$. It seems from this figure that all the peaks in the pattern are due to the PET substrate. No peak was detected for the as-deposited SnO thin film, which shows that the film is an amorphous structure. Raman spectroscopy was applied to confirm the phase formation of SnO thin film, from which the characteristic vibration modes $\left(\mathrm{A}_{1 \mathrm{~g}}\right.$ and $\left.\mathrm{B}_{1 \mathrm{~g}}\right)$ are detected as shown in Fig. 2. The two strong modes in Fig. 2 have frequencies about 107 and $212 \mathrm{~cm}^{-1}$ correspond to $B_{1 \mathrm{~g}}$ symmetry and $\mathrm{A}_{1 \mathrm{~g}}$ symmetry modes, respectively, which are in agreement with others [17-20]. The phase purity of the prepared sample is confirmed by the high Raman spectrum intensity. No other peaks have been detected for $\mathrm{SnO}_{2}$ or other intermediate oxides [19]. AFM is used to investigate the topography (unevenness and roughness) of the obtained $\mathrm{SnO}$ thin film. Figure 3 displays the two-dimensional (2D) and three-dimensional (3D) AFM images of SnO/PET thin film. From the 2D AFM image, it is observed that the deposited film shows a granular morphology texture. The 3 AFM D image shows that the surface of $\mathrm{SnO}$ thin film is formed topographically from hills and valleys which are distributed uniformly over the entire film surface. From the images, the root mean square roughness $\left(\mathrm{R}_{\mathrm{q}}\right)$ can be estimated, it is found equal to $6.387 \mathrm{~nm}$. The average particle size of the $\mathrm{SnO}$ thin film is in the range of $(\sim 80 \mathrm{~nm})$. Also, the values of the maximum roughness valley depth $\left(\mathrm{R}_{\mathrm{V}}\right)$ are found equal to $70.75 \mathrm{~nm}$ and mean maximum roughness valley depth $\left(\mathrm{R}_{\mathrm{vm}}\right)$ is found equal to $28.32 \mathrm{~nm}$.

Fig. 1 XRD pattern of the $\mathrm{SnO} / \mathrm{PET}$ thin film

Fig. 2 Raman spectrum of the $\mathrm{SnO} / \mathrm{PET}$ thin film
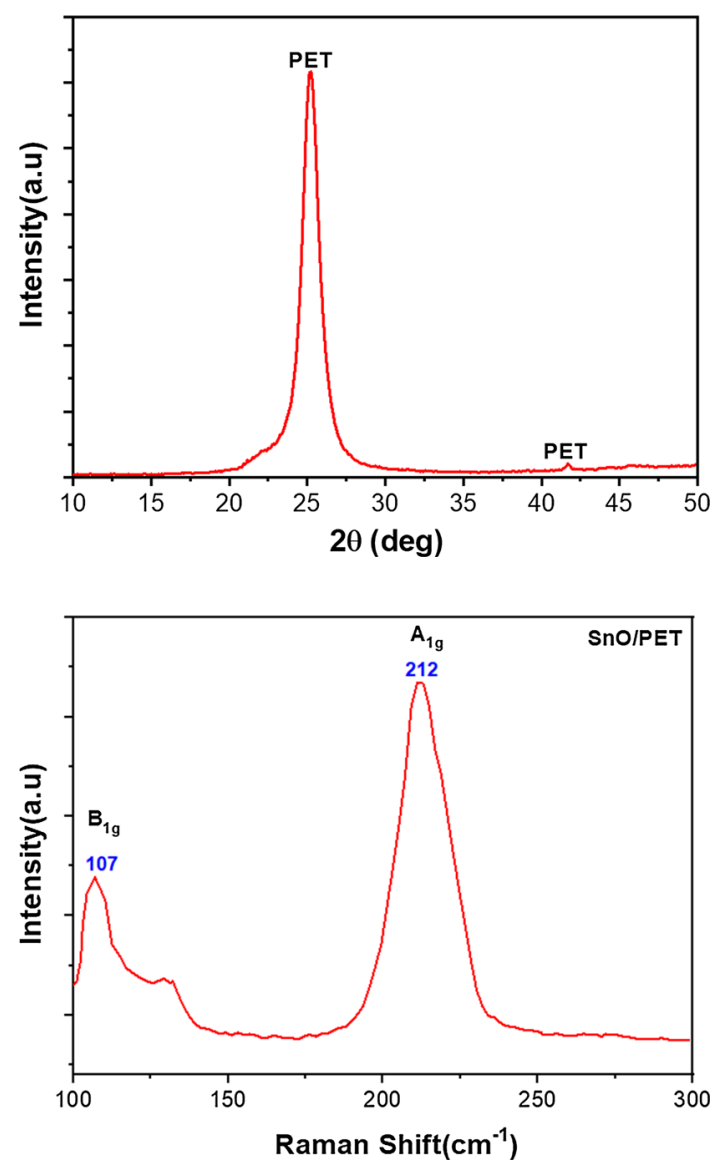

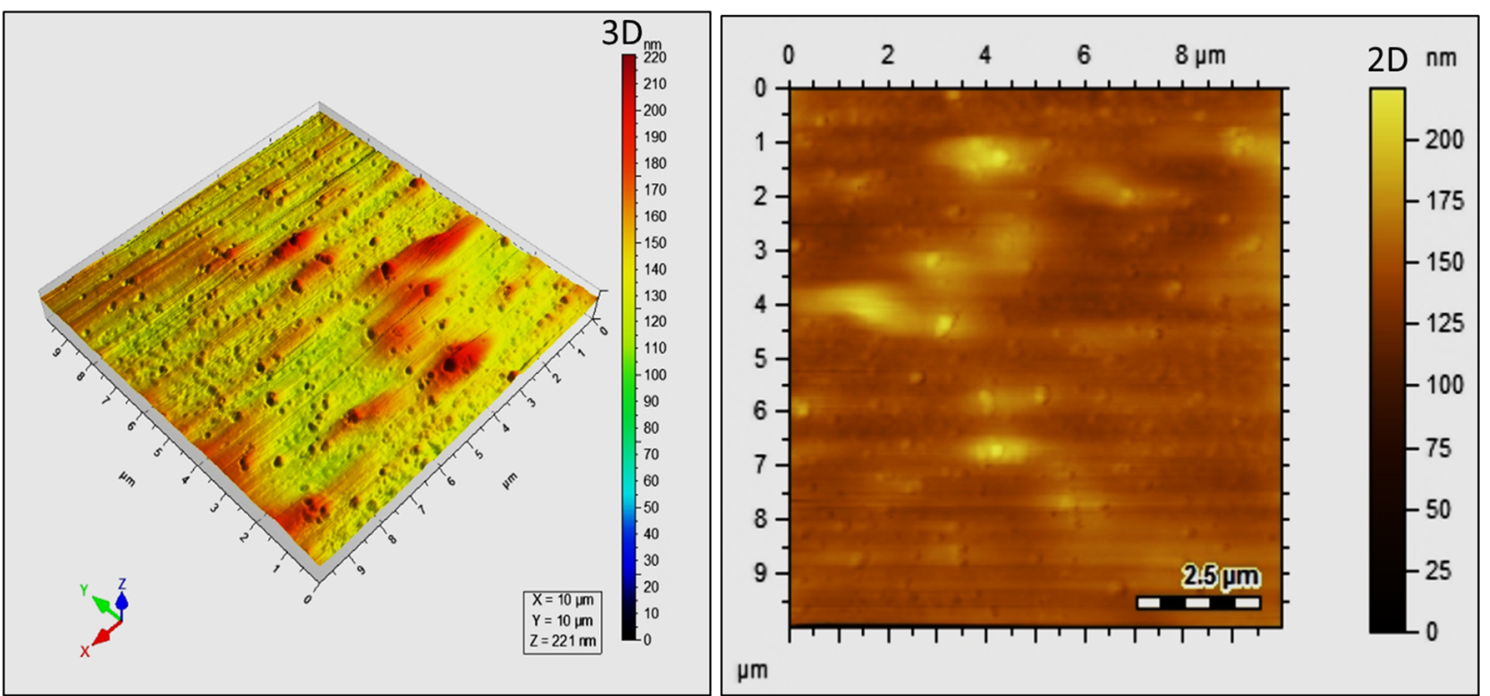

Fig. 3 The 2D\&3D AFM image for SnO/PET thin film

Fig. 4 The $T(\lambda)$ and $R(\lambda)$ spectra for $\mathrm{SnO} / \mathrm{PET}$ thin film

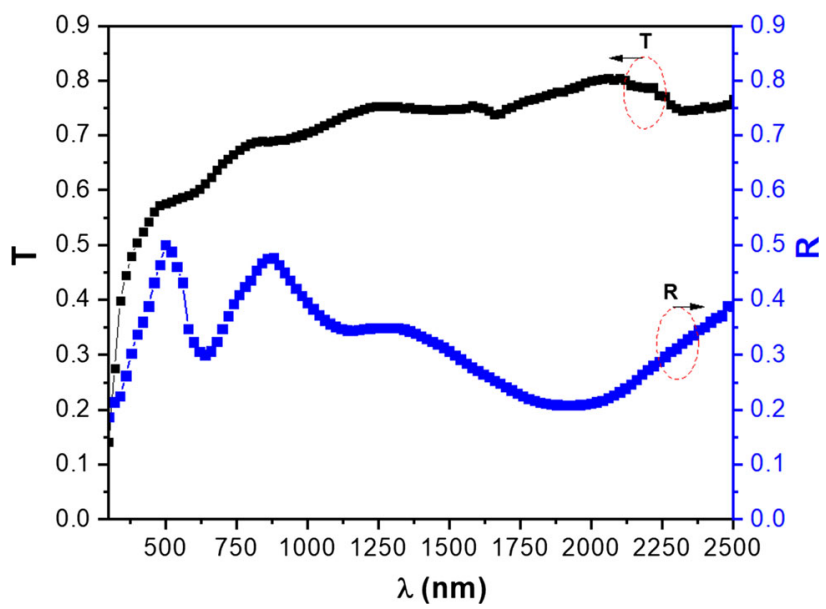

\subsection{The linear optical properties of SnO/PET}

Figure 4 shows the variation of $\mathrm{T}(\lambda)$ and $\mathrm{R}(\lambda)$ of as-deposited $\mathrm{SnO} / \mathrm{PET}$ thin film at wavelength range $300-2500 \mathrm{~nm}$. It could be observed that the film displays a high transmittance (T>70\%) for the all wavelength range. Moreover, it is clear that $\mathrm{T}(\lambda)$ is mostly limited by the PET substrate. Higher roughness like the case of the PET substrate would cause lighter scattering that may lead to higher reflectance. The spectrum pattern of a semiconductor's absorption coefficient $\alpha$ at the fundamental edge is of high interest for the study of the allowed transitions in addition to the structure of the energy band. The absorption coefficient of SnO/PET thin film is valued from the $T(\lambda) \& R(\lambda)$ data using the following equation [17]:

$$
\alpha=\frac{1}{d} \ln \left[\frac{(1-R)^{2}}{2 T}+\sqrt{\frac{(1-R)^{4}}{4 T^{2}}+R^{2}}\right]
$$

The $\alpha$ curve for SnO/PET thin film is shown in Fig. 5a, from which it is noted that the absorbance spectrum of SnO thin film exhibits an increase in the absorbance in the range less than $400 \mathrm{~nm}$. This behavior indicates the transparency of SnO thin film in the visible region. The absorbance spectra of the SnO thin film, in the near-infrared region (NIR) region (700-2500 nm), take low values and are nearly constant with increasing the wavelength. The optical band gap, $\mathrm{E}_{\mathrm{g}}^{\mathrm{Opt}}$ for SnO thin film, in the absorption region, can be estimated by Tauc's equation [21-24]:

$$
\alpha \mathrm{hv}=B\left(h v-E_{\mathrm{g}}^{\mathrm{Opt}}\right)^{r}
$$

where, hv is the photon energy, and $\mathrm{E}_{\mathrm{g}}^{\mathrm{Opt}}$ is the optical band gap, $\mathrm{B}$ is a constant, $\mathrm{r}$ is an index depending on the transition probability, and optical transition type which takes $(\mathrm{r}=0.5)$ for direct allowed transitions. The $\mathrm{E}_{\mathrm{g}}^{\mathrm{Opt}}=3.49 \mathrm{eV}$ for SnO thin film which is obtained 
Fig. 5 a Absorption coefficient, $\alpha$ and $\mathbf{b}$ tauc plot for SnO/PET thin film
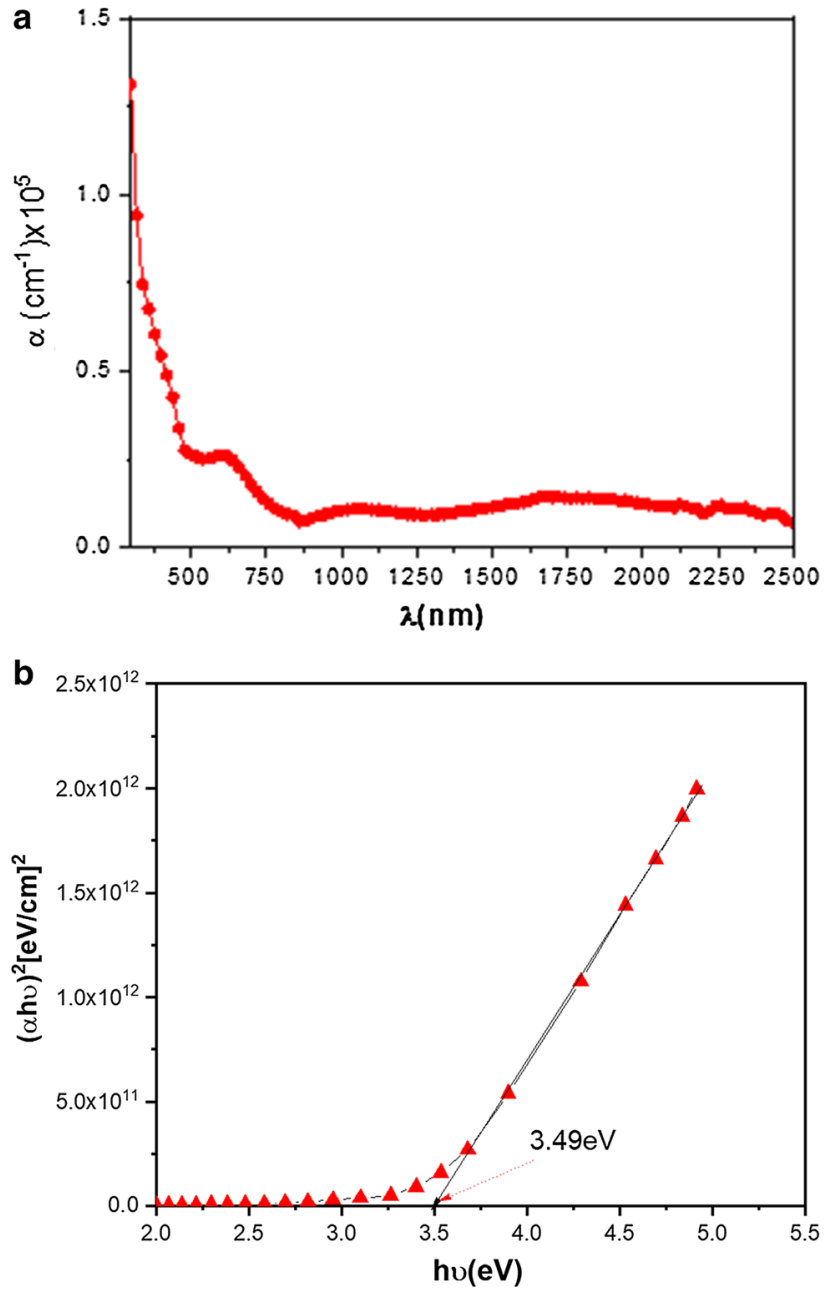

from plotting $(\alpha \mathrm{h} v)^{1 / 2}$ vs. (hv) as shown in Fig. 5b. The estimated $E_{\mathrm{g}}^{\mathrm{Opt}}$ is agreed with the previous reports for the amorphous band gap for $\mathrm{SnO}$ thin film $[5,17,25,26]$. For a semiconducting material to be applicable in optical and optoelectronic applications such as solar cells and optical fibers, the determination of optical constants such as refraction index (n) and extinction coefficient (k) is essential. The refractive index is a fundamental characteristic of the material that can be employed in many designs and applications like optoelectronic devices, laser limiting, photovoltaic, and other waveguide devices. The refractive index (n) can be calculated by using Fresnel's relation [17, 24]:

$$
\mathbf{n}=\frac{1+\boldsymbol{R}}{1-\boldsymbol{R}}+\sqrt{\frac{4 \boldsymbol{R}}{(1-\boldsymbol{R})^{2}}-\boldsymbol{k}^{2}}
$$

where, $\mathbf{k}$ is the extinction coefficient, which is calculated by the next equation [24]:

$$
k=\frac{\alpha \lambda}{4 \pi}
$$

Figure 6 displays the spectral distribution of $\mathbf{k}(\boldsymbol{\lambda})$ and $\mathbf{n}(\boldsymbol{\lambda})$ for SnO thin film deposited on to the PET substrates. Extinction coefficient $\mathbf{k}(\boldsymbol{\lambda})$ is beneficial in determining the lost light quantity due to scattering. In Fig. 6 , $\mathbf{k}(\boldsymbol{\lambda})$ displays an absorption peak in the visible range until $750 \mathrm{~nm}$. While, at wavelengths $(750-2000 \mathrm{~nm}), \mathbf{k}(\lambda)$ shows an absorption peak with a wide hump. Moreover, it is obvious that in Fig. 6, $\mathrm{n}(\lambda)$ is dependent on the wavelength. In the range of 300-2500 $\mathrm{nm}, \mathbf{n}(\lambda)$ shows two peaks at 500 and $850 \mathrm{~nm}$. Then, $\mathbf{n}(\lambda)$ decreases with the wavelength increase (normal dispersion). At normal dispersion, $\mathbf{n}(\lambda)$ for the $\mathrm{SnO} / \mathrm{PET}$ thin film can be exanimated by the WDD single oscillator model [27]:

$$
n^{2}(h v)=1+\frac{E_{d} E_{o}}{E_{o}^{2}-(h v)^{2}}
$$

where, $\boldsymbol{E}_{\boldsymbol{d}}$ is the average of dispersion energy optical transitions, and $E_{o}$ is the single-oscillator energy for the electronic excitations. Figure 7 shows the plotting of $\left(\boldsymbol{n}^{2}-1\right)^{-1}$ vs. $(\boldsymbol{h} \boldsymbol{v})^{2}$ for SnO/ PET thin film. The values of $\boldsymbol{E}_{\boldsymbol{d}}$ and $\boldsymbol{E}_{\boldsymbol{o}}$ can be calculated from the 
Fig. 6 The spectral distribution of the optical constant $\mathbf{a} \mathrm{k}$ and $\mathbf{b} \mathrm{n}$, for $\mathrm{SnO} / \mathrm{PET}$ thin film

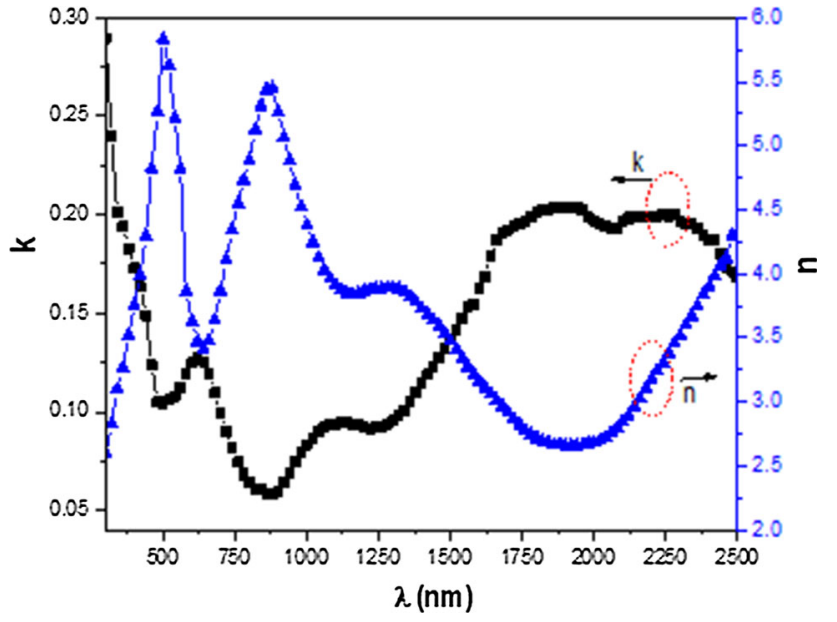

Fig. 7 Variation of $\left(n^{2}-1\right)^{-1}$ as a function of $(h v)^{2}$ for $\mathrm{SnO} / \mathrm{PET}$ thin film

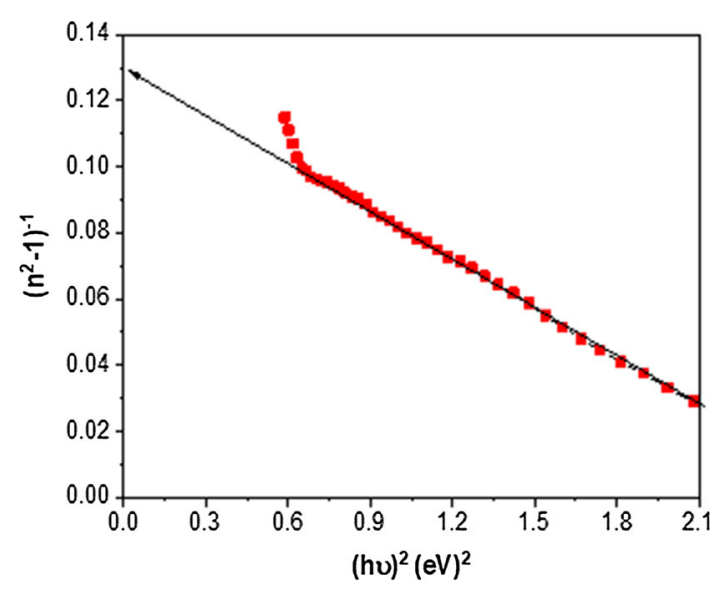

slope and intercept [28] in Fig. 7. Furthermore, the constant of the optical dielectric at a higher frequency $\left(\boldsymbol{\varepsilon}_{\infty}\right)$ is determined by the following formula:

$$
\varepsilon_{\infty}=\left(1+\frac{E_{d}}{E_{o}}\right)^{0.5}
$$

The obtained values of WDD dispersion parameters $\left(\boldsymbol{E}_{\boldsymbol{o}}, \boldsymbol{E}_{\boldsymbol{d}}\right.$ and $\left.\boldsymbol{\varepsilon}_{\infty}\right)$ for SnO/ PET thin film are 1.63, 12.3, and 8.56 eV, respectively. In a transparent region, the impact of lattice vibration modes of dispersion and the free carriers is as given in the following relation [29]:

$$
n^{2}=\varepsilon_{L}-\left(\frac{e^{2}}{4 \pi^{2} c^{2} \varepsilon_{o}}\right)\left(\frac{N}{m^{*}}\right) \lambda^{2}
$$

where, $\varepsilon_{\mathrm{L}}$ is the high-frequency dielectric constant, $e$ is the electron charge, $\varepsilon_{o}$ is the free space permittivity, $c$ is the light velocity, and $\left(\frac{N}{m^{*}}\right)$ is the ratio of carrier concentration to the effective mass. Figure 8 shows the variation of $n^{2}$ with $\lambda^{2}$. The values of $\varepsilon_{L}$ and $\frac{N}{m^{*}}$ are obtained from the intercept $\left(\lambda^{2}=0\right)$ and the slope of Fig. 8. The resulted values of $\varepsilon_{L}$ and $\frac{\mathrm{N}}{\mathrm{m}^{*}}$ are 19.66 and 4.62 $\times 10^{57} \mathrm{~m}^{-3} \mathrm{Kg}^{-1}$, respectively. The values of $\varepsilon_{\mathrm{L}}$ are higher than $\varepsilon_{\infty}$ which can be owing to the free charge carrier influence in the polarization process that is occurred within the material through exposure to light [30].

The polarizability of a material that relies on the density of states inside the prohibited gap can be expressed by the dielectric constant. The real part and imaginary part (dielectric constant, $\boldsymbol{\varepsilon}_{\mathbf{1}}$, and dielectric loss, $\boldsymbol{\varepsilon}_{\mathbf{2}}$ ) for SnO thin film can be estimated by the following relations [17, 30]:

$$
\begin{gathered}
\varepsilon_{1}=n^{2}-k^{2} \\
\varepsilon_{2}=2 n k
\end{gathered}
$$

The variations of $\varepsilon_{1}$ and $\varepsilon_{2}$ for SnO/PET are displayed in Fig. 9, from which it is clear that $\varepsilon_{1}$ and the refractive index have similar patterns, while the dielectric loss $\varepsilon_{2}$ and the extinction coefficient have the same design. The reduction in $\boldsymbol{\varepsilon}_{\mathbf{1}}$ can be regarded as an improvement in the optical response of the $\mathrm{SnO}$ thin film. This increment in polarizability (optical response) can be attributed to the 
Fig. $8 \mathrm{n}^{2}$ as a function of $\lambda^{2}$ for $\mathrm{SnO} / \mathrm{PET}$ thin film

Fig. 9 The variation of $\varepsilon_{1}$ and $\varepsilon_{2}$ vs. $\lambda$ for SnO/PET thin film
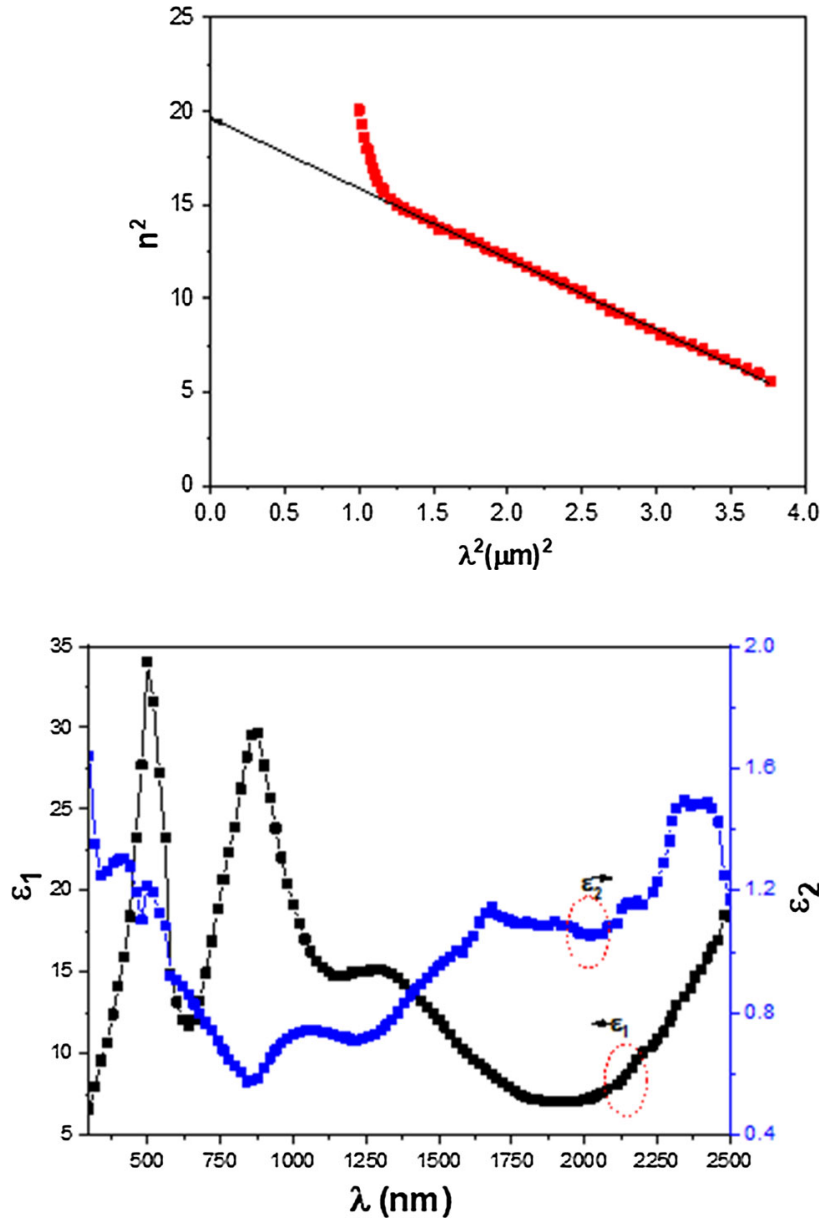

increase in the disorder degree and, therefore, the rise in the density of states in the forbidden gap. In the analysis of the energy loss rate for electrons passing through the surface and volume of material, the essential values of the real and imaginary portions of the complex dielectric constant components are determined through the volume energy loss functions (VELF) and surface energy loss (SELF). The energy loss rate for electrons travelling through the surface and volume of material using the VELF and SELF can be calculated using the values $\varepsilon_{1}$ and $\varepsilon_{2}$ as follows [17]:

$$
\begin{gathered}
\text { VELF }=-\operatorname{lm}\left(\frac{1}{\varepsilon^{*}}\right) \frac{\varepsilon_{2}}{\left(\varepsilon_{1}^{2}+\varepsilon_{2}^{2}\right)} \\
\mathrm{SELF}=-\operatorname{lm}\left(\frac{1}{\varepsilon^{*}+1}\right) \frac{\varepsilon_{2}}{\left(\left(\varepsilon_{1}^{2}+1\right)^{2}+\varepsilon_{2}^{2}\right)}
\end{gathered}
$$

From Fig. 10, it is obvious that the variation of VELF and SELF with wavelengths (750-2500 nm) is significant. In the bulk material, the energy loss of the free charge carriers has almost identical behavior when they cross the surface, particularly for SnO/PET thin films with lower energies. There is also no significant difference between them at the lower and higher photon energies except that VELF rises more than SELF at the unique peak that characterizes the lower and greater photon energies.

\subsection{The nonlinear optical parameters for SnO/PET thin film}

Nonlinear optics (NLO) has had a significant influence on technology since it has assisted in the expansion of the photonics field. Thin-film NLO features were investigated because of their relevance in applications such as capacity communications and photonic switching. Polarizability $\mathrm{P}$ in the material is expressed as in the following relations [31-33]:

$$
P=\chi^{(1)} E+\chi^{(2)} E^{2}+\chi^{(3)} E^{3}
$$


Fig. 10 The variation of VELF and SELF vs. $\lambda$, for SnO/PET thin film

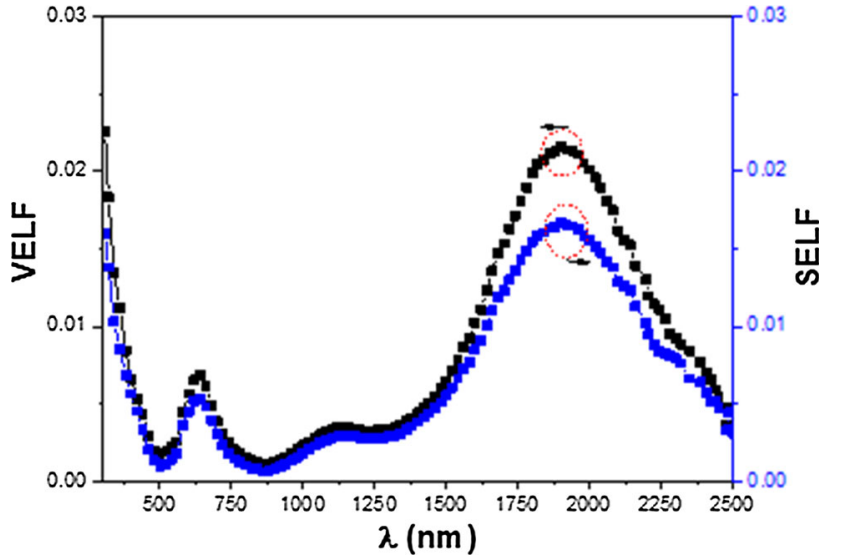

where, $\chi^{(\mathbf{1})}$ is the linear optical susceptibility, while $\chi^{(\mathbf{2})}$ and $\chi^{(\mathbf{3})}$ are the second- and third-order NLO susceptibilities, which can provide extensive data about using thin films in optoelectronic applications. The $\chi^{(\mathbf{1})}$ and $\chi^{(3)}$ can be given in the following expression [17, 34-37]:

$$
\begin{gathered}
\chi^{(1)}=\frac{n^{2}-1}{4 \pi} \\
\chi^{(3)}=1.7\left[\chi^{(1)}\right]^{4} \times 10^{-10} \\
\chi^{(3)}=1.7\left[\frac{n^{2}-1}{4 \pi}\right]^{4} \times 10^{-10}
\end{gathered}
$$

The nonlinear refractive index $\boldsymbol{n}^{(2)}$ also related to the third-order nonlinear optics $\boldsymbol{\chi}^{(3)}$ and the static refractive index $\boldsymbol{n}_{0}$ can be estimated by the following equation [36].

$$
n^{(2)}=\frac{12 \pi \chi^{(3)}}{n_{o}}
$$

The static refractive index $\left(\mathbf{n}_{\mathbf{0}}\right)$ is calculated by the formula $\left(\mathbf{n}_{\mathbf{0}}=\sqrt{\boldsymbol{\varepsilon}_{\infty}}\right)$. Figure 11a shows the variation in $\chi^{(\mathbf{1})}$ vs. hv for SnO thin film, and Fig. 11b displays the change in $\chi^{(\mathbf{3})}$ and $\boldsymbol{n}^{(2)}$ values with hv. The optical switching capability of SnO/PET thin film with noticeable peaks between 1.42 and $2.47 \mathrm{eV}$ is shown in Fig. $11(\mathrm{a} \& \mathrm{~b}) . \chi^{(3)}$ has maximum values of $3.88 \times 10^{-10}$ and $4.51 \times$ $10^{-10} \mathrm{esu}$ for SnO/PET thin-films with photon energies of 1.42 and $2.47 \mathrm{eV}$, respectively. While $n^{(2)}$ values for SnO/PET thin films are $5.16 \times 10^{-9}$ and $5.95 \times 10^{-9}$ at photon energies of 1.42 and $2.47 \mathrm{eV}$, respectively.

The third-order nonlinear optical characteristics of SnO nanoparticles (3.7 nm) were investigated by Ara et al. [38], they discovered that $\mathrm{SnO}$ nanoparticles had nonlinear refractive indices in the range of $10^{-7} \mathrm{~cm}^{2} / \mathrm{W}$ with a negative sign. Yu et al. [39] assessed the $\chi^{(3)}$ of $\mathrm{SnO}_{2}$ nanoparticles in both naked and coated with stearic acid. These findings demonstrated that these nanoparticles had substantially bigger $\chi^{(3)}$ than the bulk material. Because the quantum confinement effect confines electron mobility in nanoparticles to a three-dimensional infinite-depth potential well, the electronic cloud is distorted and the high-frequency Kerr effect is amplified when incoming light is used. Moreover, Endo et al. [40] discovered that the oxygen concentration in amorphous a-SnO ${ }_{2-\mathrm{x}}$ thin films was intermediate between that of crystalline c-SnO and $\mathrm{c}-\mathrm{SnO}_{2}$, and that decreasing the oxygen content of films increased $\chi^{(3)}$ values. Where, the assessed values of $\chi^{(3)}$ for a- $\mathrm{SnO}_{2-\mathrm{x}}$ were in the range of $6 \times 10^{-12}$ to $16.6 \times 10^{-12}$ esu, which were several orders of magnitude greater than that for $\mathrm{c}-\mathrm{SnO}_{2-\mathrm{x}}\left(3.2 \times 10^{-12} \mathrm{esu}\right)$. The origin of significant $\chi^{(3)}$ and $\boldsymbol{n}^{(2)}$ for tin oxide compounds is owing to the high mobility of optically stimulated electrons in the conduction band, which is composed of spherically symmetric $5 \mathrm{~s}$ orbitals of $\mathrm{Sn}$ atoms and contributes to dipole moment enlargement produced by optical stimulation. The reduction in oxygen concentration and particle sizes also had a role in the large $\chi^{(\mathbf{3})}$ [38-42].

Finally, high values of $\chi^{(3)}$ and $n^{(2)}$ suggest that the SnO/PET thin film is commonly used in nonlinear optical systems, such as optical limiters and photonic devices. One of the most essential mechanisms for inducing nonlinear absorption $\beta \mathrm{c}$ in materials is the two-photon absorption TPA. This phenomenon happens in materials when the incoming light's energy is greater than $E_{\mathrm{g}}^{\mathrm{Opt}} / 2 \mathrm{for}$ thin films. The $\beta \mathrm{c}$ is calculated (see Fig. 12.) according to straightforward empirical formula developed by Sheik-Bahae et al. [43-45]. The $\beta \mathrm{c}$ grows as hv increases until it hits a maximum $\left(\beta_{\text {cmax }}=4.6 \mathrm{~cm} / \mathrm{GW}\right.$ at $\left.2.47 \mathrm{eV}\right)$ at which point it decreases again. A similar response of $\beta c$ with hv was discovered in several metal oxides, including $\mathrm{PbO}_{2} /$ glass [46], $\mathrm{MnO}_{2} /$ glass [47], and $\mathrm{BiOI} / \mathrm{FTO}$ [48]. According to our previous work [17], the $\beta_{\text {cmax }}=4.36 \mathrm{~cm} / \mathrm{GW}, \beta_{\text {cmax }}=8.67 \mathrm{~cm} / \mathrm{GW}$ and $\beta_{\text {cmax }}=13.85 \mathrm{~cm} / \mathrm{GW}$ for SnO$/ \mathrm{glass}$, $\mathrm{SnO} / \mathrm{FTO}$, and $\mathrm{SnO} / \mathrm{ITO}$, respectively. Clearly, the difference in $\beta_{\text {cmax }}$ values is attributable to the effect of substrates. 
Fig. 11 The variation of $\mathbf{a} \chi^{(1)}$, $\mathrm{SnO} / \mathrm{PET}$ thin film b $\chi^{(3)}$, and $n^{(2)}$ vs. hv for
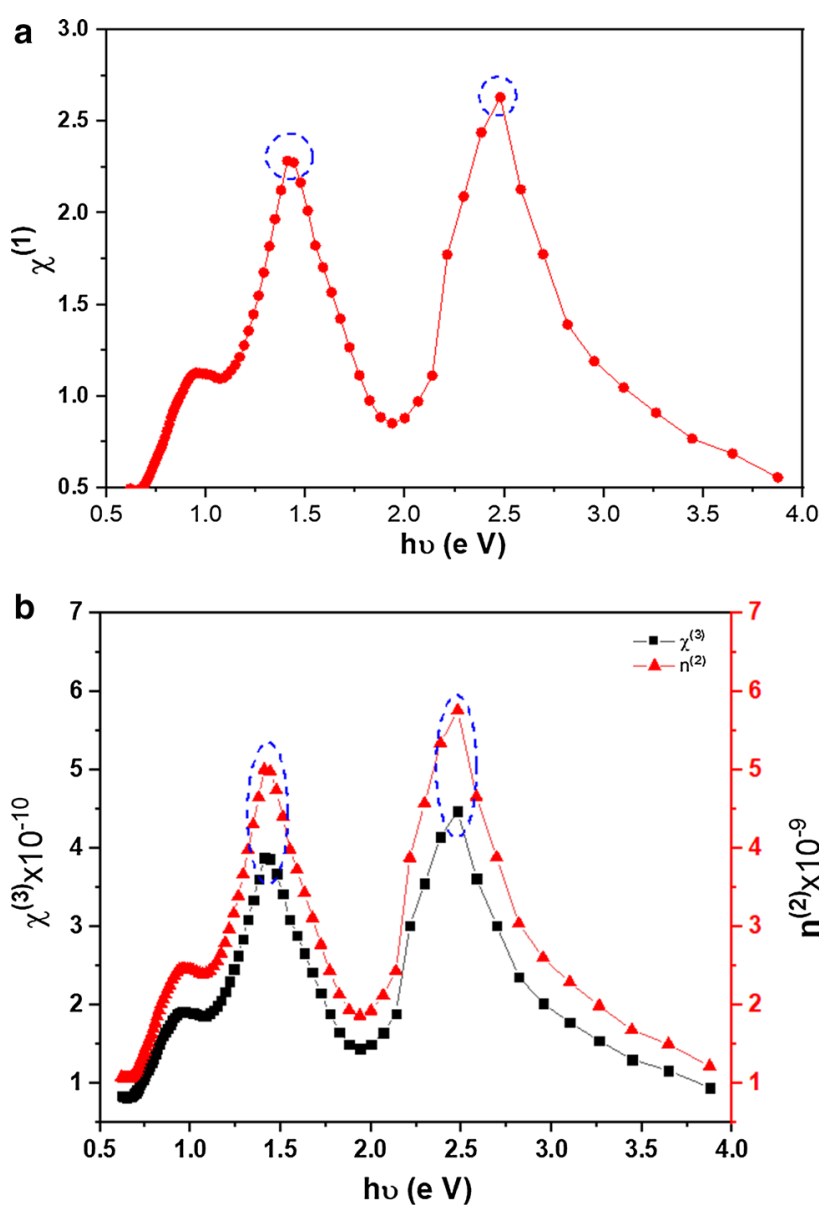

Fig. 12 The variation of $\beta c$ vs. hv for SnO/PET thin film

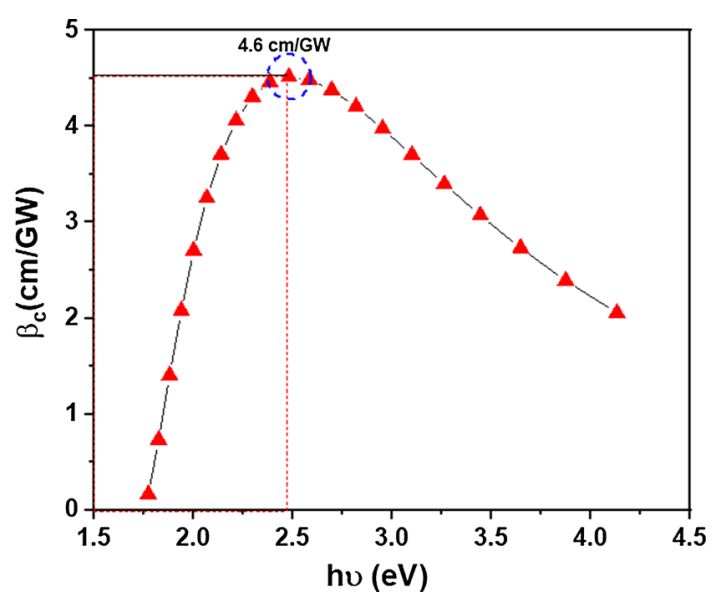

\section{Conclusions}

In conclusion, we have studied the morphological, structural, and optical properties of flexible SnO (II) deposited on to a PET substrate via thermal evaporation deposition. The XRD pattern of the SnO/PET thin film indicated that the SnO thin film was an amorphous structure. Raman spectroscopy was applied to confirm the phase formation of the SnO thin film. The AFM image showed that the surface of the $\mathrm{SnO}$ thin film was topographically formed from hills and valleys that are distributed uniformly over the entire film surface. The direct value was about $3.49 \mathrm{eV}$. The refractive index for the SnO/PET thin film obeyed the single oscillator model at the normal dispersion region. Finally, the nonlinear characteristics of the SnO/PET thin film demonstrated magnificent switching behavior, indicating that the SnO/PET thin film might be used in optical switching devices operating at photon energies between 
1.42 and $2.47 \mathrm{eV}$. It was determined that $\beta_{c \max }=4.6 \mathrm{~cm} / \mathrm{GW}$ using the formula presented by Sheik-Bahae et al. These findings indicate that $\mathrm{SnO} / \mathrm{PET}$ thin film will be a promising candidate for flexible optical system applications.

Funding Open access funding provided by The Science, Technology \& Innovation Funding Authority (STDF) in cooperation with The Egyptian Knowledge Bank (EKB).

Data Availability Statement This manuscript has associated data in a data repository. [Authors' comment: All data included in this manuscript are available upon request by contacting the corresponding author.]

Open Access This article is licensed under a Creative Commons Attribution 4.0 International License, which permits use, sharing, adaptation, distribution and reproduction in any medium or format, as long as you give appropriate credit to the original author(s) and the source, provide a link to the Creative Commons licence, and indicate if changes were made. The images or other third party material in this article are included in the article's Creative Commons licence, unless indicated otherwise in a credit line to the material. If material is not included in the article's Creative Commons licence and your intended use is not permitted by statutory regulation or exceeds the permitted use, you will need to obtain permission directly from the copyright holder. To view a copy of this licence, visit http://creativecommons.org/licenses/by/4.0/.

\section{References}

1. K. Ide, K. Nomura, H. Hosono, T. Kamiya, Electronic defects in amorphous oxide semiconductors: a review. Phys. Status Solidi A 216, 1800372 (2019)

2. L. Petti, N. Münzenrieder, C. Vogt, H. Faber, L. Büthe, G. Cantarella, F. Bottacchi, T.D. Anthopoulos, G. Tröster, Metal oxide semiconductor thin-film transistors for flexible electronics. Appl. Phys. Rev. 3, 021303 (2016)

3. C. Zhang, G. Liu, X. Geng, K. Wu, M. Debliquy, Metal oxide semiconductors with highly concentrated oxygen vacancies for gas sensing materials: a review. Sens. Actuat. A. Phys. 309, 112026 (2020)

4. B. Jayant Baliga, Wide Band gap Semiconductor Power Devices, Woodhead Publishing, Sawston (2019).

5. L.Y. Liang, Z.M. Liu, H.T. Cao, Z. Yu, Y.Y. Shi, A.H. Chen, H.Z. Zhang, Y.Q. Fang, X.L. Sun, Phase and optical characterizations of annealed SnO thin films and their p-type TFT application. J. Electrochem. Soc. 157, H598 (2010)

6. T. Yang, J. Zhao, X. Li, X. Gao, C. Xue, Y. Wu, R. Tai, Preparation and characterization of p-type transparent conducting SnO thin films. Mater. Lett. 139, $39(2015)$

7. A. Diallo, E. Manikandan, V. Rajendran, M. Maaza, Physical \& enhanced photocatalytic properties of green synthesized SnO2 nanoparticles via Aspalathus linearis. J. Alloys Compd. 681, 561 (2016)

8. W. Guo, L. Fu, Y. Zhang, K. Zhang, L.Y. Liang, Z.M. Liu, H.T. Cao, X.Q. Pan, Microstructure, optical, and electrical properties of p-type SnO thin films. Appl. Phys. Lett. 96, 042113 (2010)

9. H. Chen, X. Wang, L. Guan, L. Chen, J. Tao, Surface engineering of layered SnO micro-plates for impressive high supercapacitor performance. Mater. Chem. Phys. 238, 121889 (2019)

10. H.-S. Jeong, M.-J. Park, S. Hun Kwon, H.-J. Joo, S.-H. Song, H. In Kwon, Low temperature NO2 sensing properties of RF sputtered $\mathrm{SnO}^{-S n O}{ }_{2}$ heterojunction thin-film with p-type semiconducting behavior. Ceram. Int. 44, 17283 (2018)

11. P. Jiang, J. Jing, Y. Wang, H. Li, X. He, Y. Chen, W. Liu, Facilely transforming bulk materials to SnO/pristine graphene 2D-2D heterostructures for stable and fast lithium storage. J. Alloys Compd. 812, 152114 (2020)

12. S. Seal, S. Shukla, Nanocrystalline SnO gas sensors in view of surface reactions and modifications. JOM. 54, 35 (2002)

13. R. Martins, A. Nathan, R. Barros, L. Pereira, P. Barquinha, N. Correia, R. Costa, A. Ahnood, I. Ferreira, E. Fortunato, Complementary metal oxide semiconductor technology with and on paper. Adv. Mater. 23, 4491 (2011)

14. F.F. Vidor, T. Meyers, U. Hilleringmann, Flexible electronics: Integration processes for organic and inorganic semiconductor-based thin-film transistors. Electronics 4, 480-506 (2015)

15. E.F.M. El-Zaidia, A.A.A. Darwish, I.S. Yahia, M, Rashad, Noncrystalline films of gallium (III) phthalocyanine chloride evaporated on a flexible polymer substrate for flexible organic technology: optical spectroscopy and optical limiting. Phys. Scr. 95, 115802 (2020)

16. A.A.A. Darwish, A.M. Aboraia, A.V. Soldatov, I.S. Yahia, Deposition of Rhodamine B dye on flexible substrates for flexible organic electronic and optoelectronic: optical spectroscopy by Kramers-Kronig analysis. Opt. Mater. 95, 109219 (2019)

17. A.A. Abuelwafa, M.S. Abd El-sadek, S. Elnobi, T. Soga, Effect of transparent conducting substrates on the structure and optical properties of Tin (II) oxide (SnO) thin films: Comparative study. Ceram. Int. 47, 13510 (2021)

18. Y.A. El-Gendy, Effects of film thickness on the linear and nonlinear refractive index of p-type SnO films deposited by e-beam evaporation process. Physica B. 526, 59 (2017)

19. B. Eifert, M. Becker, C.T. Reindl, M. Giar, L. Zheng, A. Polity, Y. He, C. Heiliger, P.J. Klar, Raman studies of the intermediate tin-oxide phase. Phys. Rev. Mater. 1, 014602 (2017)

20. K.M. Abhirami, R. Sathyamoorthy, K. Asokan, Structural, optical and electrical properties of gamma irradiated SnO thin films. Radiat. Phys. Chem. 91, 35 (2013)

21. J. Tauc, A. Menth, States in the gap. J. Non-Cryst. Solids 8, 569 (1972)

22. T.S. Aldeen, H. Elsayed, A. Mohamed, M. Maaza, Bio-inspired Single Phase Monteponite cdo nanoparticles via natural extract of phoenix roebelenii palm leaves. J. Inorg. Organomet. Polym. Mater. 30, 4691 (2020)

23. A.C. Nwanyaa, R. Deshmukh, R.U. Osuji, M. Maaza, C.D. Lokhande, F.I. Ezema, Synthesis, characterization and gas-sensing properties of SILAR deposited $\mathrm{ZnO}-\mathrm{CdO}$ nano-composite thin film. Sens. Actuat. B. 206, 671 (2015)

24. A.A. Abuelwafa, M.S. Abd El-sadek, I.S. Yahia, Linear and nonlinear optical properties of nano-spherical Perylenetetracarboxylic dianhydride/ITO as a new optical system. Opt. Laser Technol. 108, 241 (2018)

25. R. Sivaramasubramaniam, M.R. Muhamad, S. Radhakrishna, Optical properties of annealed tin (11) oxide in different ambients. Phys. Stat. Sol. 136, $215(1993)$

26. E. Eqbal, E.I. Anila, Properties of transparent conducting tin monoxide (SnO) thin films prepared by chemical spray pyrolysis method. Physica B. 528, $60(2018)$

27. S.H. Wemple, M. DiDomenico, Behavior of the electronic dielectric constant in covalent and ionic materials. Phys. Rev. B. 3, 1338-1351 (1971)

28. R.M.D. Matiura, A.A. Abuelwafa, S. Kato, N. Kishi, T. Soga, A comparative study on optical properties of $\mathrm{BiOI}_{1} \mathrm{Bi}_{7} \mathrm{O}_{9} \mathrm{I}_{3}$ and $\mathrm{Bi}_{5} \mathrm{O}_{7} \mathrm{I}$ materials. Opt. Mater. 111, 110677 (2021) 
29. M. Dongol, A.F. Elhady, M.S. Ebied, A.A. Abuelwafa, Effect of thermal annealing on the optical properties of thin films. Indian. J. phys. 95, 1245-1253 (2021)

30. A.A. Abuelwafa, A. El-Denglawey, M. Dongol, M.M. El-Nahass, T. Soga, Structural and optical properties of nanocrystalline platinum octaethylporphyrin (PtOEP) thin films. J. Alloys Compd. 655, 415-422 (2016)

31. M. Frumar, J. Jedelsky, B. Frumarov, T. Wagner, M. Hrdlicka, Optically and thermally induced changes of structure, linear and non-linear optical properties of chalcogenides thin films. J. Non. Cryst. Solid. 326 \& 327, 399 (2003)

32. A. Abdel-Galil, M.A. Assiri, I.S. Yahia, Optical analysis of methyl violet thin flms/polymeric substrate for fexible organic technology. Opt. Quantum Electron. 52, $377(2020)$

33. H. Nasu, J. Matsuoka, K. Kamiya, Second- and third-order optical non-linearity of homogeneous glasses. J. Non-Cryst. Solids 178, 23 (1994)

34. H. Tichá, L. Tichý, Semiempirical relation between non-linear susceptibility (refractive index), linear refractive index and optical gap and its application to amorphous chalcogenides. J. Optoelectron. Adv. Mater. 4, 381 (2002)

35. H. Nasu, J.D. Mackenzie, Nonlinear optical properties of glasses and glass- or gel-based composites. Opt. Eng. 26, 102 (1987)

36. H.M. Alsoghier, M.A. Selim, H.M.A. Salman, H.M. Rageh, M.A. Santos, S.A. Ibrahim, M. Dongol, T. Soga, A.A. Abuelwafa, NMR spectroscopic, linear and non-linear optical properties of 1,3-benzothiazol-2-yl-(phenylhydrazono)acetonitrile (BTPA) azo dye. J. Mol. Struct. 1179, 315 (2019)

37. A.A. Abuelwafa, H.M. Alsoghier, S. Elnobi, M. Dongol, T. Soga, Quantum computational, linear and non-linear optical properties of spin-coated nickel (II)-tetraphenylporphyrin/FTO thin films. Optik 234, 166618 (2021)

38. M.H.M. Ara, P. Boroojerdian, Z. Javadi, S. Zahedi, M. Morshadian, Nonlinear optical properties of ultra-fine nanocrystalline SnO synthesised through microwave-assisted hydrothermal route. Micro Nano Lett. 6, 249 (2011)

39. B.-L. Yu, G.-L. Zhang, G.-Q. Tang, X.-C. Wu, W.-J. Chen, B.-Z. Yang, P. Zhao, Third-order nonlinear optical properties of SnO $\mathrm{S}_{2}$ nanoparticles. Acta Phys. Sin. 5, 377 (1996)

40. H. Endo, M. Sakai, Y. Watanabe, M. Takata, Third-order nonlinear optical susceptibilities of amorphous $\mathrm{SnO}_{2-\mathrm{x}}$ thin films. Appl. Phys. Lett. 62, 559 (1993)

41. H.P. Asha, N.B. Gummagol, P.S. Patil, B.V. Rajendra, Modification of structure, electrical, linear and third-order nonlinear optical properties of spray pyrolyzed tin oxide films by deposition temperature. Superlatt. Microstruct. 155, 106920 (2021)

42. Y. Watanabe, M. Sakai, M. Takata, N. Ueda, H. Kawazoe, Third order nonlinear optical susceptibility for transparent tin oxide thin films. Ferroelectrics 131, $111(1992)$

43. S. Bahae, D.C. Hutchings, D.J. Hagan, E.W.V. Stryland, Dispersion of bound electron nonlinear refraction in solids. IEEE J. Quantum Electron. 27, $1296(1991)$

44. M. Sheik-Bahae, D.J. Hagan, E.W. Van Stryland, Dispersion and band-gap scaling of the electronic Kerr effect in solids associated with two-photon absorption. Phys. Rev. Lett. 65, 96 (1990)

45. A.A. Abuelwafa, S. Elnobi, I. Yamada, N. Shibata, T. Soga, Studying linear and nonlinear optical properties of trifluoroethoxy-coated zinc phthalocyanine ((4TFEO) 4-ZnPc) thin films. Opt. Mater. 123, 111850 (2022)

46. H.M. Zeyada, M.M. Makhlouf, Role of annealing temperatures on structure polymorphism, linear and nonlinear optical properties of nanostructure lead dioxide thin films. Opt. Mater. 54, 181 (2016)

47. M.M. Makhlouf, Preparation and optical characterization of $\beta-\mathrm{MnO}_{2}$ nano thin films for application in heterojunction photodiodes. Sens. Actuat. 279, $145(2018)$

48. A.A. Abuelwafa, R.M.D. Matiur, A.A. Putri, T. Soga, Synthesis, structure, and optical properties of the nanocrystalline bismuth oxyiodide (BiOI) for optoelectronic application. Opt. Mater. 109, $110413(2020)$ 